\title{
Role of Helicobacter pylori in ulcer healing and recurrence of gastric and duodenal ulcers in longterm NSAID users. Response to omeprazole dual therapy
}

\author{
G Bianchi Porro, F Parente, V Imbesi, F Montrone, I Caruso
}

\begin{abstract}
Background-The relation between Helicobacter pylori infection and nonsteroidal anti-inflammatory drug (NSAID)-associated peptic ulcers remains unclear; in particular, it is not known whether $H$ pylori plays a part in the healing and recurrence of these ulcers.

Aims-To evaluate prospectively in a consecutive series of arthritis patients receiving longterm NSAID treatment the prevalence of peptic ulcer as well as the effect of $H$ pylori eradication on the healing and recurrence of gastric and duodenal ulcer found.

Patients-Some 278 consecutive patients underwent gastroscopy with multiple biopsies of the gastric antrum and corpus for histological examination and rapid urease test. One hundred peptic ulcers (59 gastric ulcers, 39 duodenal ulcers, and two gastric ulcers concomitant with a duodenal ulcer) were found. Seventy per cent of these ulcers were $H$ pylori positive. Methods-According to their $H$ pylori status, ulcer patients were randomised to one of the following treatments: $H$ pylori negative ulcers received omeprazole 20 mg twice daily for four to eight weeks, whereas $H$ pylori positive lesions were treated with omeprazole $20 \mathrm{mg}$ twice daily plus amoxycillin $1 \mathrm{~g}$ twice daily (the second of these for the first two weeks) or omeprazole alone for four to eight weeks while continuing NSAID therapy. Patients with healed ulcers were endoscopically followed up for six months after stopping antiulcer therapy while continuing NSAIDs.
\end{abstract}

Results-Endoscopic healing rates for gastric and duodenal ulcers in the three different groups were similar both at four and eight weeks. $H$ pylori eradication did not influence healing, which occurred in 14 of $20(70 \%)$ of patients in whom $H$ pylori was eradicated, compared with 14 of 17 $(82 \%)$ of patients with persistent infection. Cumulative recurrence rates at six months did not statistically differ among the three different groups $(27 \%$ in $H$ pylori negative, $46 \%$ in $H$ pylori positive, and $31 \%$ in those where $H$ pylori was eradicated during the healing phase), although a numerical trend in favour of a higher recurrence rate in infected patients was evident.
Conclusions-H pylori eradication does not confer any significant advantage on the healing of gastric and duodenal ulcers associated with longterm NSAID use. It remains to be established with certainty whether eradication may be helpful in the reduction of recurrence in a specific subset of NSAID associated ulcer.

(Gut 1996; 39: 22-26)

Keywords: $H$ pylori, NSAIDs, peptic ulcers, arthritis.

Although Helicobacter pylori infection and nonsteroidal anti-inflammatory drugs (NSAIDs) are commonly considered the most important exogenous factors in the aetiology of peptic ulcer disease, little is known about the relation between these two agents in patients with NSAID associated peptic ulcers. ${ }^{1}$

Indeed, very few prospective studies have dealt with the possible interaction between NSAIDs and $H$ pylori on the clinical course of NSAID induced gastroduodenal lesions, yielding conflicting results. Lanza et $a l^{2}$ found that $H$ pylori infection has no influence on the development of gastroduodenal mucosal lesions after the short-term administration of naproxen or aspirin in healthy volunteers. Similarly, Kim et $a l^{3}$ showed that $H$ pylori infection does not confer increased risk of ulceration in arthritic patients receiving NSAIDs in the long term. In contrast, Taha et al $^{4}$ showed that peptic ulcers in longterm NSAID users are more likely to develop in the presence of $H$ pylori infection and duodenal erosions. To date, no prospective study has evaluated whether the eradication of $H$ pylori might influence the healing process of peptic ulcer induced by NSAIDs. In addition, no firm data are yet available on peptic ulcer recurrence in arthritic patients who receive NSAIDs daily after initial healing, although Seppala et al have recently shown that the eradication of $H$ pylori seems to reduce gastric ulcer recurrences even in patients taking NSAIDs more than once a week. ${ }^{5}$ The question is not irrelevant if we consider that at least $50 \%$ of gastroduodenal ulcers in longterm NSAID users are associated with $H$ pylori infection ${ }^{367}$ and, therefore, they might benefit particularly from an antibacterial treatment if a synergistic relation between this bacterium and NSAID could be confirmed.

Therefore, we have aimed at investigating prospectively the effect of $H$ pylori eradication 
on healing and recurrence of NSAID associated peptic ulcers in a group of arthritic patients receiving NSAIDs in the long term.

\section{Methods}

Patients

Patients aged 18 years or over were recruited from the Rheumatology Outpatients' Clinic provided that they had osteoarthritis, adult rheumatoid arthritis, psoriatic arthritis, ankylosing spondylitis or Reiter's syndrome. Daily NSAID therapy had to have been taken for at least four weeks before baseline endoscopy and was expected to continue for at least an additional six months. Patients were excluded if they had had gastric surgery, gastrointestinal malignancy, or if they had taken antiulcer agents or antibiotics within three weeks of endoscopy. Other exclusion criteria included pyloric obstruction, recent acute upper gastrointestinal bleeding, and severe renal impairment. In addition, patients taking anticoagulants, cytotoxic drugs or prednisone at dosages $>10$ $\mathrm{mg} /$ day were excluded. Demographic data including age, sex, smoking habits, alcohol consumption, and history of ulcer disease were specifically looked for and recorded.

Informed consent was obtained at baseline endoscopy, which was performed using diazepam for sedation. An ulcer was defined as a localised defect in gastric or duodenal mucosa of at least $5 \mathrm{~mm}$ in diameter and with perceptible depth, whereas smaller lesions were considered as erosions. Ulcer dimensions were measured using standard Olympus biopsy forceps, with the fully open spoon being equivalent to $5 \mathrm{~mm}$.

\section{Study design}

$H$ pylori was determined by a biopsy urease test and histological assessment. For this purpose, apart from specimens from gastric ulcer edges, three biopsy specimens were taken from the antrum and two from the corpus. One antral biopsy specimen was used for the rapid urease test (CP test, Brocades, Milan, Italy), whereas the remaining formalin fixed specimens were used for histological assessment. The specimens were embedded in paraffin wax and stained with haematoxylin and eosin and modified Giemsa methods. Gastric histopathology was classified according to a personal modification of the Sydney system as previously described. ${ }^{8}$ A patient was considered $H$ pylori positive when $H$ pylori was found at histological examination and rapid urease test was positive.

Patients with active gastric or duodenal ulcer at initial endoscopy were asked to participate in a prospective trial to evaluate the effect of $H$ pylori on ulcer healing and recurrence. The presence of concurrent gastric or duodenal erosions did not constitute a criterion for exclusion from the trial.

According to their $H$ pylori status they were assigned to the following ulcer healing treatments: $H$ pylori positive patients received omeprazole $20 \mathrm{mg}$ twice daily for four weeks and amoxycillin $1 \mathrm{~g}$ twice daily during the first two weeks or omeprazole $20 \mathrm{mg}$ twice daily alone for four weeks according to a randomised single blind scheme. $H$ pylori negative patients were all treated with omeprazole $20 \mathrm{mg}$ twice daily for four weeks. Patients also continued to take NSAIDs at the same dose given prior to the study during both the ulcer healing and ulcer recurrence phases of the study. Patient compliance to antiulcer treatment was determined by counting all the unused tablets reported by the patients at fortnightly clinical assessments; during these visits side effects were specifically asked for and recorded.

After four weeks patients were endoscoped again to assess ulcer healing; if the ulcer was still active they received an open therapy with omeprazole $20 \mathrm{mg}$ twice daily for a further four weeks.

Patients were considered to have been cured of $H$ pylori if both rapid urease test and histological examination in the antrum and corpus were negative. In patients whose ulcer had healed at four weeks, $H$ pylori eradication was assessed after one month from stopping treatment; in patients whose ulcer healed at eight weeks, because of the possibility of a false negative result at this time while receiving omeprazole, $H$ pylori status was taken on the basis of the result of biopsies done at three months during the follow up. Similarly, in patients with unhealed ulcẹr after eight weeks, $H$ pylori status was re-evaluated one to three months afterwards while they were receiving alternative therapy (sucralfate, misoprostol) after stopping NSAIDs.

Patients with healed ulcers at eight weeks, irrespective of their $H$ pylori status, were asked to participate in the second part of the study. During this phase patients continued to take NSAIDs at the same dose given during the ulcer healing phase for a further six months but stopped any antiulcer therapy. Endoscopy was repeated at 12 and 24 weeks of follow up or before in the case of a recurrence of ulcer symptoms, with the endoscopist unaware of the $H$ pylori status of the patients. Patients who took at least $85 \%$ of the antiulcer treatment, who missed no more than two consecutive days of NSAID therapy, and who had both the second and third endoscopic examination were considered evaluable for the first end point of the study.

Any patient who took at least $70 \%$ of NSAID therapy and did not miss more than three consecutive days of treatment during the longterm phase was considered evaluable for the second end point of the study.

Statistical analysis included the Student's $t$ test for unpaired samples, and the $\chi^{2}$ and Fisher's exact test where appropriate. Ninety five per cent confidence intervals for the difference in healing and recurrence rates between groups were also calculated.

\section{Results}

A total of 278 consecutive arthritic patients taking longterm NSAID treatment underwent 
TABLE I Demographic and clinical characteristics of the evaluable patients in relation to the presence of a gastric or duodenal ulcer

\begin{tabular}{lll}
\hline Characteristic & Ulcer & No ulcer \\
\hline Patients (n) & 100 & 146 \\
Age (y) (mean) & 59 & 56 \\
Sex & $78(78)$ & $120(82)$ \\
Women (\%) & $22(22)$ & $26(18)$ \\
Men (\%) & $52(52)$ & $76(52)$ \\
Gastric erosions present (\%) & $28(28)$ & $32(22)$ \\
Duodenal erosions present (\%) & $34(34)$ & $25(17)^{\star}$ \\
History of ulcer disease (\%) & $24(24)$ & $36(25)$ \\
Arthritic condition & $68(68)$ & $98(67)$ \\
Osteoarthritis (\%) & $8(8)$ & $12(8)$ \\
Rheumatoid arthritis (\%) & $70(70)$ & $72(49)^{\star}$ \\
Other (\%) & $30(30)$ & $74(51)$ \\
H pylori status & & \\
Positive (\%) & & \\
Negative (\%) & & \\
\hline *p $<0.01$. &
\end{tabular}

diagnostic upper gastrointestinal endoscopy in our unit over the study period, and 246 of these satisfied the inclusion criteria and were therefore recruited into the study.

Some 142 of these patients (58\%) had $H$ pylori infection identified by histology and rapid urease test. At initial endoscopy, 59 patients had gastric ulcer alone, 39 duodenal ulcer alone, and two both duodenal and gastric ulcer. Table I shows the demographic and clinical characteristics of these patients compared with those without ulcers. Patients in both groups were comparable with respect to their age, sex, smoking and drinking habits, number of gastric or duodenal erosions, and type of arthritic condition, whereas a previous history of ulcer disease and $H$ pylori infection was significantly more frequent in the ulcer than in the non-ulcer group. In contrast, no significant difference in the types, dose or length of NSAID intake was found between the two groups.

\section{Site and size of gastric ulcers}

Most ulcers (53 (83\%) of 64 ) were located at the gastric antrum; seven $(11 \%)$ occurred in the angulus area and four (6\%) in another part of the corpus. The mean ulcer size, $6 \mathrm{~mm}$ (range 5-15) was similar in all three study groups.

\section{Ulcer healing phase}

Of the 70 patients with $H$ pylori positive gastric or duodenal ulcer, 36 received omeprazole 20 mg twice daily for four to eight weeks and

TABLE II $\mathrm{H}$ pylori and healing of gastric and duodenal ulcers

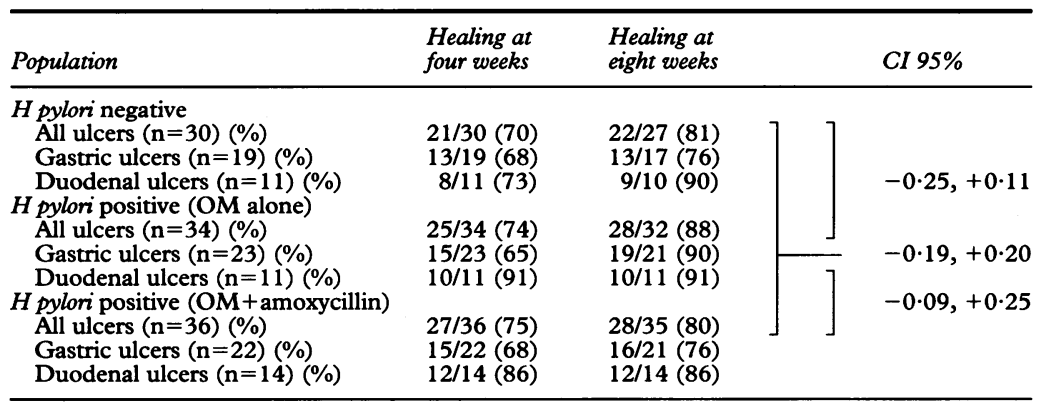

$\mathrm{OM}=$ omeprazole
TABLE III $\mathrm{H}$ pylori and recurrence of NSAID associated gastric or duodenal ulcer

\begin{tabular}{|c|c|c|}
\hline Population & $\begin{array}{l}\text { Cumulative recurrence } \\
\text { at six months }\end{array}$ & CI 95\% \\
\hline $\begin{array}{l}\text { Group A }(\mathrm{n}=19)(\%) \\
\text { (H pylori negative) }\end{array}$ & $5 / 18(27)$ & \multirow{3}{*}{$\begin{array}{l}-0.48,+0.10 \\
-0.36,+0.28 \\
-0.17,+0.28\end{array}$} \\
\hline $\begin{array}{l}\text { Group B }(\mathrm{n}=29)(\%) \\
(\text { H pylori positive) }\end{array}$ & $13 / 28(46)$ & \\
\hline $\begin{array}{l}\text { Group C }(\mathrm{n}=14)(\%) \\
\text { (H pylori eradicated) }\end{array}$ & $4 / 13(31)$ & \\
\hline
\end{tabular}

amoxycillin $1 \mathrm{~g}$ twice daily during the first two weeks, whereas the remaining 34 were treated with omeprazole $20 \mathrm{mg}$ twice daily alone for four to eight weeks. All the 30 patients with $H$ pylori negative gastric or duodenal ulcer received omeprazole $20 \mathrm{mg}$ twice daily for four to eight weeks. Table II shows the healing rates for gastric and duodenal ulcers in the three different groups at four and eight weeks of treatment. There were no significant differences in the percentages of healing between the different groups of treatment for both gastric and duodenal ulcers.

$H$ pylori infection was cured in 20 of 36 $(56 \%)$ patients who received the antibiotic regimen and in none of those treated with omeprazole alone but did not significantly influence the healing process. Indeed, healing occurred in 14 of 20 patients $(70 \%)$ who were cured of $H$ pylori and in 14 of $17(82 \%)$ patients with persistent infection.

Eleven patients with gastric ulcer and five with duodenal ulcer were still unhealed after eight weeks of treatment. However, in both groups the maximum ulcer diameter significantly reduced during treatment, thus suggesting a slow response rather than resistance to treatment. Indeed, for these gastric ulcers the mean diameter fell from 7 (before therapy) to $3.6 \mathrm{~mm}$ (at eight weeks) with a $49 \%$ reduction and, similarly, for the duodenal ulcers, it reduced from 6.2 to $3.5 \mathrm{~mm}$ corresponding to a reduction of $42 \%$.

\section{Ulcer recurrence phase}

A total of 62 patients with healed peptic ulcer after eight weeks of acute antiulcer treatment entered this phase of the study. They were subdivided into three different groups according to their baseline $H$ pylori status: 19 patients were $H$ pylori negative (group A), 29 patients had persistent $H$ pylori infection after short-term therapy (group B) whereas 14 patients had been cured of $H$ pylori after the short-term phase (group C). Fifty nine patients completed the study correctly and were therefore evaluable: 18 in group A, 28 in group $B$, and 13 in group C. Three patients defaulted: one in group $A$ failed to attend the three month endoscopic control, another in group B refused to have another endoscopy at six months, and one in group C did not adhere strictly to NSAID treatment. Table III shows the cumulative recurrence rate at six months for the three different groups. There were no significant differences between groups although a numerical trend towards a lower rate of recurrence was evident in $H$ pylori negative and patients cured of $H$ pylori 
compared with those with persistent $H$ pylori infection.

\section{Discussion}

Despite the fact that it is now well accepted that NSAIDs and $H$ pylori have distinct pathogenic roles in peptic ulcer disease, the relation between these two factors remains unclear. ${ }^{1}$ Current evidence seems to show that the presence of $H$ pylori infection does not constitute a significant additional risk factor for NSAID gastropathy, ${ }^{23679-11}$ but it is suspected that NSAID use exacerbates $H$ pylori ulcers and therefore the existence of a small group of patients whose ulcers results from an interaction between $H$ pylori and NSAIDs could be hypothesised. This is indirectly supported by a study that showed that peptic ulcers in longterm NSAID users are more likely to develop in patients with $H$ pylori positive duodenal erosions. ${ }^{4}$

Whether or not $H$ pylori and NSAIDs have a synergistic relation in NSAID associated ulcers can be best considered by a prospective study evaluating the role of eradicating $\mathrm{H}$ pylor $i$ in the healing and recurrence of these ulcers such as reported here. In a large population of arthritic patients taking longterm NSAID treatment we found a prevalence of longterm gastric or duodenal ulceration of $36 \%$ (100 of 278). This is consistent with previous studies performed by us and by other authors reporting that NSAID associated peptic damage occurs in approximately $30 \%$ of NSAID users. ${ }^{12}{ }^{13}$ Fifty eight per cent of the patients studied were $H$ pylori positive, which is what can be expected in a Western control population of similar age and in line with the data previously reported by others in arthritic patients. ${ }^{3714}$

Most $(70 \%)$ NSAID associated ulcers were associated with $H$ pylori infection and antral gastritis; this concurs well with that seen by Shallcross et $a l^{6}$ who showed that in ulcer patients taking NSAIDs, approximately three quarters are $H$ pylori positive. However, the fact that as many as $30 \%$ of these ulcers occurred in uninfected patients strengthens the theory that these drugs exert an ulcerogenic effect that does not necessarily require the presence of $H$ pylori or gastritis. ${ }^{15}$

One of the most important results of the ulcer healing phase of our study was that the cumulative healing at eight weeks, especially for gastric ulcers, was fairly poor (approximately $80 \%$ ), considering that these patients were all receiving $40 \mathrm{mg}$ omeprazole per day. This finding differs from that seen by Walan et $a^{16}$ in a multicentre trial comparing ranitidine with omeprazole in gastric ulcers, where omeprazole seemed more effective. However, in the second study NSAIDs were used simply on demand and not daily as for our patients. Our results, therefore, suggest that irrespective of the cause, the regular use of NSAIDs delays ulcer healing even with omeprazole treatment and not only under treatment with less potent agents (ranitidine) as already reported. ${ }^{17} 18$

Unlike non-NSAID associated peptic ulcers where healing is increased by $H$ pylori eradica- tion, ${ }^{19}$ we found that $H$ pylori infection has not a clear influence on healing of NSAID associated peptic ulcers. Indeed, healing rates of both duodenal and gastric ulcers did not significantly differ between patients with persistent $H$ pylori infection and those in whom the organism had been eradicated. On the other hand, it should be emphasised that the $H$ pylori cure rate obtained with omeprazole/ amoxycillin in these patients (that is $56 \%$ ) is comparable with that we and others have reported in non-NSAID related peptic ulcers using the same combination. ${ }^{19-22}$

In addition, we showed that $H$ pylori eradication did not significantly reduce the frequency of recurrence in patients who continued to receive longterm NSAID treatment but stopped antiulcer medications. Unfortunately, the comparatively small number of ulcers (mainly duodenal ulcers) included in the longterm phase of our study does not completely exclude the possibility that $H$ pylori infected patients taking NSAIDs would not have an increased risk of developing ulcer recurrence compared with $H$ pylori negative patients; this is particularly true if we consider the existence of a numerical trend but not statistically significant in favour of a higher recurrence rate in infected compared with uninfected patients or those cured from the infection and the fact that we did not compare each one of the two common causes of ulcer with both together. The question could have been better tackled by including a third control population constituted by $H$ pylori positive healed ulcers not receiving NSAID in the longterm phase.

However, the finding that eradication of $H$ pylori was not associated with the cure of NSAID associated peptic ulcers suggest that the additional risk induced by the presence of $H$ pylori, if any, tends to be small and eventually its eradication would be worthwhile only in a subset of patients receiving longterm NSAID treatment. The results of this study, if confirmed, may have important consequences in clinical practice as they suggest that the determination of $H$ pylori status in a patient with NSAID associated gastric or duodenal ulcer does not provide any relevant additional information concerning ulcer healing; in contrast, it remains to be established, in further studies including larger numbers of $H$ pylori infected and uninfected patients with NSAID associated peptic ulcers, whether a subset of ulcers caused by both agents does exist and whether these ulcers might benefit in the long run from $H$ pylori eradication.

1 Taha AS, Russel RI. Helicobacter pylori and non-steroidal anti-inflammatory drugs: uncomfortable partners in peptic ulcer disease. Gut 1993; 34: 580-3.

2 Lanza DS, Evans DG, Graham DY. Effect of Helicobacter pylori infection on the severity of gastroduodenal mucosa injury after the acute administration of naproxen or aspirin in normal volunteers. Am $\mathcal{F}$ Gastroenterol 1991; 86: 735-7.

3 Kim JG, Graham DY and The Misoprostol Study Group. Helicobacter pylori infection and development of gastric Helicobacter pylori infection and development of gastric
or duodenal ulcer in arthritic patients receiving chronic or duodenal ulcer in arthritic patients receiving chro
NSAID therapy. Am $f$ Gastroenterol 1994; 89: 203-7.

4 Taha AS, Sturrock RD, Russel RI. Mucosal erosions in the longterm non-steroidal anti-inflammatory drug users: predisposition to ulceration and relation to Helicobacter pylori. Gut 1995; 36: 334-6. 
5 Seppala JK, Pikkarainen P, Sipponen P, Kivilaakso E, Gormesen NH and The Finnish gastric ulcer study group. Cure of peptic gastric ulcer associated with eradication of H pylori. Gut 1995; 36: 834-7.

6 Shallcross TM, Rathbone BJ, Wyatt II, Heatley RV. Helicobacter pylori associated chronic gastritis and peptic Helicobacter pylori associated chronic gastritis and peptic ulceration in patients taking non-steroidal anti-inflam-
matory drugs. Aliment Pharmacol Therap 1990; 4: matory

7 Iaine L, Marin-Sorensen M, Weinstein WM. Nonsteroidal antiinflammatory drug-associated gastric ulcers do not require Helicobacter pylori for their development. $\mathrm{Am} \mathcal{F}$ Gastroenterol 1992; 87: 1398-402.

8 Solcia E, Villani L, Fiocca R, Luinetti O, Trespi E, Perego $M$, et al. Effects of eradication of helicobacter pylori in duodenal ulcer patients. Scand $¥$ Gastroenterol 1994; 29 (suppl 201): 28-31.

9 Loeb DS, Talley NJ, Ahlquist DA, Carpenter HA, Zinmeister AR. Long-term nonsteroidal anti-inflammatory drug use and gastroduodenal injury: the role of tory drug use and gastroduodenal injury: the role of
Helicobacter pylori. Gastroenterology 1992; 102: Helicobacter

10 Graham DY, Lidsky MD, Cox AM, Doyle JE, Evans DG, Alpert $\mathrm{L}$, et al. Long-term nonsteroidal anti-inflammatory drug use and. Helicobacter pylori infection. Gastroenterology 1991; 100: 1653-7.

11 Soll AH, Weinstein WM, Kurata J. Nonsteroidal antiinflammatory drugs and peptic ulcer disease. Ann Intern Med 1991; 114: 307-19.

12 Caruso I, Bianchi Porro G. Gastroscopic evaluation of antiinflammatory agents. $B M \mathcal{F} 1980 ; 280: 75-8$.

13 McCarthy DM. Nonsteroidal antiinflammatory druginduced ulcers: management by traditional therapies. induced ulcers: management by
Gastroenterology 1989; 96: 662-74.
14 Taha AS, Capell HA, Sturrock RD, Russel RI. Non steroidal peptic damage in rheumatoid patients receiving second-line drugs. Am $\mathcal{F}$ Gastroenterol 1991; 86: 1588-91.

$15 \mathrm{McCarthy} \mathrm{DM}$. Helicobacter pylori infection and gastroduodenal injury by non-steroidal anti-inflammatory drugs. Scand $\mathcal{F}$ Gastroenterol 1991; 26 (suppl 187): 91-7.

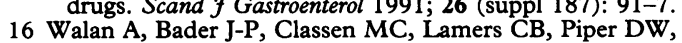
Rutgersson $\mathrm{K}$, et al. Effect of omeprazole and ranitidine Rutgersson $\mathrm{K}$, et al. Effect of omeprazole and ranitidine
on ulcer healing and relapse rates in patients with benign on ulcer healing and relapse rates in patients
gastric ulcer. $N$ Engl $\mathcal{Y}$ Med 1989; 320: 69-75.

17 Manniche C, Malchow-Moller A, Andersen JR, Pedersen C, Hansen TM, Jess P, et al. Randomised study of the influence of non-steroidal antiinflammatory drugs on the treatment of peptic ulcer in patients with rheumatic disease. Gut 1987; 28: 226-9.

18 Lancaster-Smith MJ, Jadeberg ME, Jackson DA. Ranitidine in the treatment of non-steroidal anti-inflammatory drug gastric and duodenal ulcer. Gut 1991; 32. 252-5.

19 Graham DY, Lew GM, Evans DJ, Klein PD. Effect of triple therapy (antibiotic plus bismuth) on duodenal ulcer healtherapy (antibiotic plus bismuth) on duodenal ulcer heal115: $266-9$.

20 Axon ATR. The role of acid inhibition in the treatment of $\mathbf{H}$ pylori infection. Scand $\mathcal{f}$ Gastroenterol 1994;29 (suppl 201): 16-23.

21 Tytgat GNJ. Review article: treatment that impact favourably upon eradication of $\mathrm{H}$ pylori and ulcer recurrence. Aliment Pharmacol Therap 1994; 8: 359-68.

22 Parente F, Maconi G, Bargiggia S, Colombo E, Bianchi Porro G. Comparison of two different lansoprazole-antibiotic combinations for the eradication of $H$ pylori infection in duodenal ulcer patients. Aliment Pharmacol Therap 